\title{
Rab25 is responsible for phosphoinositide 3-kinase/AKT-mediated cisplatin resistance in human epithelial ovarian cancer cells
}

\author{
YANG FAN $^{1}$, LONG WANG $^{2}$, XUECHUAN HAN $^{1}$, XUEQIN LIU $^{1}$ and HONGYUN MA ${ }^{1}$ \\ ${ }^{1}$ Department of Obstetrics and Gynecology, Ningxia People's Hospital, Yinchuan, Ningxia 750000; \\ ${ }^{2}$ Department of Stomatology, Ningxia Medical University, Yinchuan, Ningxia 750004, P.R. China
}

Received January 24, 2014; Accepted September 12, 2014

DOI: $10.3892 / \mathrm{mmr} .2014 .2963$

\begin{abstract}
Rab25, a member of the Rab family of small guanosine triphosphatase, was reported to have an essential role in the development of human epithelial ovarian cancer. The present study demonstrated that Rab25 mediated the sensitivity of ovarian cancer to cisplatin, a first-line chemotherapeutic agent for the treatment of ovarian cancer in the clinic. Overexpression of Rab25 and increased phosphoinositide 3-kinase (PI3K)/AKT signaling were detected in cisplatin-resistant SKOV-3 cells compared with those in cisplatin-sensitive ES-2 cells. The results of the present study indicated that cisplatin resistance was primarily due to reduced G1 cell cycle arrest following cisplatin treatment in SKOV-3 cells. By contrast, the corresponding phenomenon was not observed following treatment with a Rab25-specific small interfering RNA or treatment with the PI3K/AKT inhibitor LY294002. Of note, inhibition of the PI3K/AKT pathway reduced Rab25 gene expression and sensitized SKOV-3 cells to cisplatin. Furthermore, knockdown of Rab25 showed an effect comparable with blocking the PI3K/AKT pathway. In conclusion, the results of the present study demonstrated that PI3K/AKT and Rab25 significantly contributed to cisplatin resistance in human epithelial ovarian cancer; in addition, silencing Rab25 or inhibiting the PI3K/AKT pathway markedly increased the sensitivity of these cells to cisplatin.
\end{abstract}

\section{Introduction}

Ovarian cancer is a malignant tumor derived from epithelial and germ cells (1). In humans, ovarian cancer is the second most prevalent gynecological malignancy and has the highest mortality rate among them (2). According to the US Centers for Disease Control and Prevention, there are $>22,000$ novel

Correspondence to: Dr Yang Fan, Department of Obstetrics and Gynecology, Ningxia People's Hospital, 301 North Zhengyuan Street, Yinchuan, Ningxia 750000, P.R. China

E-mail: fanyangys@163.com

Key words: phosphoinositide 3-kinase/AKT pathway, Rab25, cisplatin resistance, ovarian cancer cases of ovarian cancer and $\sim 14,000$ mortalities each year in the United States (3). Advanced surgical methods and chemotherapeutic agents are available for the treatment of ovarian cancer; however, early diagnosis only occurs in $\sim 25 \%$ of cases due to the lack of effective screening programs and non-specific symptoms (4-6).

Studies from the International Collaborative Ovarian Neoplasm group and the European Organization for Research and Treatment of Cancer have decided that only patients with stages IA or IB (non-clear cell histology and well-differentiated (G1) tumor; according to the International Federation of Gynecology and Obstetrics guidelines) ovarian cancer may avoid chemotherapy (7). The majority of patients with ovarian cancer require chemotherapy to enhance progression-free and overall survival. Cisplatin and analogous platinum derivatives are the front-line chemotherapeutic agents used for the management and treatment of recurrent ovarian cancer $(8,9)$.

The majority of ovarian cancers are initially responsive to chemotherapy; however, in numerous cases patients become cisplatin-resistant due to recurrence and metastasis (10). The mechanism of cisplatin resistance leading to clinical resistance remains to be elucidated. Therefore, understanding the molecular dysregulation underlying chemoresistance in ovarian cancer is essential for developing successful therapeutic strategies.

Rab25 (also known as CATX8) is a Rab11 guanosine triphosphatase (GTPase) family member that belongs to the Rab family. Rab25 was found to contain an unusual amino acid sequence, WDTAGLE, in its guanosine trisphosphate (GTP)-binding domain causing it to be constitutively activated (11). GTPase activity modulates the binding affinities of Rab25, which are critical for its biological functions, including proliferation, signal transduction, apoptosis, microtubule organization, recruitment of $\mathrm{H}^{+} \mathrm{K}^{+}$adenosine triphosphatase, transferring receptor recycling and integrin trafficking (12). Contrary to the function of other Rab11 GTPases, Rab25 overexpression correlated with the aggressiveness of cancers, including ovarian and breast cancer $(13,14)$. Studies have demonstrated that knockdown of RAB25 promoted autophagy and inhibited cell growth in ovarian cancer cells in vitro and in vivo $(15,16)$.

The aim of the present study was to investigate the effect of Rab25 overexpression and phosphoinositide 3-kinase (PI3K)/AKT signaling on cisplatin resistance in ovarian cancer cell lines in order to discover a novel strategy for sensitizing cells to chemotherapeutic agents. 


\section{Materials and methods}

Cell culture. SKOV-3 and ES-2 ovarian cancer cell lines were purchased from the Shanghai Institute of Cell Biology, Chinese Academy of Sciences (Shanghai, China). The cells were grown in RPMI 1640 medium (Gibco-BRL, Grand Island, NY, USA) and then supplemented with $100 \mathrm{IU} / \mathrm{ml}$ penicillin, $100 \mu \mathrm{g} / \mathrm{ml}$ streptomycin and $10 \%$ fetal bovine serum (FBS) (all HyClone, Logan, UT, USA) in a $5 \% \mathrm{CO}_{2}$ atmosphere at $37^{\circ} \mathrm{C}$. The cells were routinely subcultured every three days.

RNA extraction and reverse transcription quantitative polymerase chain reaction ( $R T-q P C R)$. Total cellular RNA was extracted from cells using the TRIzol ${ }^{\circledR}$ reagent (Invitrogen Life Technologies, Carlsbad, CA, USA) according to the manufacturer's instructions. Complementary DNA was synthesized using a reverse transcription reagent (Promega Corp., Madison, WI, USA). qPCR was performed using a standard SYBR green PCR kit (Promega Corp.) and PCR-specific amplification was conducted in an Eppendorf 5331 Real-Time PCR machine (Eppendorf, Hamburg, Germany). Gene expression was calculated using the $2^{-(\Delta \Delta \mathrm{Ct})}$ method. The primer sequences were synthesized by Jie Li Biology (Shanghai, China) as follows: RAB25 sense, 5'-GCCCTGGACTCTACCAATGTTGA-3' and antisense, 5'-GCTGTTCTGTCTCTGCTTGGACAC-3'; GAPDH sense, 5'-GCACCGTCAAGGCTGAGAAC-3' and antisense, 5'-TGGTGAAGACGCCAGTGGA 3'.

Western blot analysis. The cells were lysed on ice with RIPA lysis buffer [150 mM NaCl, $1 \% \mathrm{NP} 40,0.5 \%$ sodium deoxycholate, $0.1 \%$ SDS, $50 \mathrm{mM}$ Tris (pH 7.9), $10 \mathrm{mM} \mathrm{NaF}, \mathrm{PMSF}$ and $1 \mathrm{X}$ protease inhibitors], and protein concentrations were measured using the Bicinchoninic Acid Protein Assay reagent (Pierce Biotechnology, Inc., Rockford, IL, USA). Protein extracts $(40 \mu \mathrm{g})$ were subjected to SDS-PAGE and transferred onto polyvinylidene fluoride membranes (Invitrogen Life Technologies). The mouse anti-human p-AktThr308 antibody (1:1,000; Cell Signaling Technology, Inc., Danvers, MA, USA) was used as the primary antibody, and the horseradish peroxidase-labeled goat anti-mouse immunoglobulin G (1:2,000; Cell Signaling Technology, Inc.) was used as the secondary antibody. The $\beta$-actin antibody (1:5,000; Sigma, St. Louis, MO, USA) was used as an internal control. The bands were detected using an enhanced chemiluminescence kit (GE Healthcare, Little Chalfont, UK) and visualized using the ChemiDoc XRS system (Bio-Rad Laboratories, Hercules, CA, USA). Multi Gauge V3.2 software (Fujifilm, Kanagawa, Japan) was used to quantitatively determinate the gray level of each band (absorbance measured at $570 \mathrm{~nm}$ ) and the objective band/internal band ratio.

Small interfering RNA (siRNA) transfection. The human Rab25-specific siRNA (siRab25) and control siRNA (siCon) were purchased from Dharmacon, Inc. (Lafayette, CO, USA). DharmaFCET 1 reagent (Dharmacon, Inc.) was used to transfect siRNAs, according to the manufacturer's instructions. The Rab25 siRNA sense sequence used was 5'-TCCCTCTGGCTGCAGAAGT-3'.

Cell viability assay. Cells were plated into 96-well microplates $\left(10^{4}\right.$ cells/well $)$ and incubated with siRab25, siCon,
LY294002 (PI3K/AKT inhibitor; Sigma) or dimethyl sulfoxide (control; Sigma) for $24 \mathrm{~h}$. In order to investigate the inhibitory effect of cisplatin in cells, a series of cisplatin concentrations $(1,5$, 10,50 and $100 \mu \mathrm{M}$; Sigma) was added to the ovarian cancer cells. Cell viability was monitored using an MTT assay (Sigma) as previously described (17). Absorbance was measured at $570 \mathrm{~nm}$ using a Bio-Rad MicroPlate Reader, model 450 (Bio-Rad Laboratories). Experiments were performed in triplicate.

Cell cycle analysis. Cells were synchronized in G1-phase by serum starvation for $12 \mathrm{~h}$. Flow cytometric (FCM; BD FACSCalibur; Becton-Dickinson, San Jose, CA, USA) analysis was used to determine the cell cycle phases of cells. In brief, cells were washed with $4^{\circ} \mathrm{C}$ phosphate-buffered saline (PBS) and fixed with $70 \%$ cold ethanol (HyClone, Logan, UT, USA) at $4^{\circ} \mathrm{C}$ overnight. The fixed cells were then collected, washed with PBS and stained with propidium iodide (PI; Sigma) in the presence of RNAase (Sigma). The phase of cell cycle was analyzed using ModFit software, version 3.2 (Verity Software House, Topsham, ME, USA).

Apoptosis assay. To evaluate cell apoptosis, an Annexin V-fluorescein isothiocyanate (FITC) Apoptosis kit (Cell Signaling Technology, Inc.) was used. In brief, cells were harvested and washed with $4^{\circ} \mathrm{C}$ PBS. Cells were then resuspended in binding buffer and then incubated with Annexin V-FITC and PI buffers (Invitrogen Life Technologies) for $15 \mathrm{~min}$ at $4^{\circ} \mathrm{C}$ in the dark. Annexin V-FITC and PI signals were then detected using flow cytometry.

Statistical analysis. Values are presented as the mean \pm standard error of the mean. Statistical analyses were performed using Student's t-test. SPSS 17.0 software (SPSS, Inc., Chicago, IL, USA) was performed for statistical analysis $\mathrm{P}<0.05$ was considered to indicate a statistically significant difference between values.

\section{Results}

Rab25 expression and PI3K/AKT pathway activity are increased in cisplatin-resistant cell lines. The cisplatin-resistant SKOV-3 and the cisplatin-sensitive ES-2 ovarian cancer cell lines were used to investigate the role of Rab25 in cisplatin resistance in ovarian cancer. SKOV-3 cells were found to overexpress Rab25 messenger RNA (mRNA), whereas decreased expression of Rab25 mRNA was observed in the ES-2 cells (Fig. 1A). In addition, western blot analysis revealed that Rab25 protein expression levels were significantly elevated in SKOV-3 cells compared to those of ES-2 cells (Fig. 1B and C). Furthermore, AKT activity was markedly increased in the SKOV-3 cell line compared to that in the ES-2 cell line (Fig. 1B and D).

Cisplatin-resistant cell line lacks G1 cell cycle arrest following cisplatin treatment. In order to further elucidate the mechanism of cisplatin resistance in different cells, the cell cycle and apoptotic rate were analyzed. Following treatment with cisplatin for $2 \mathrm{~h}$, there was a significant dose-dependent increase in the percentage of ES-2 cells in G1-phase (Fig. 2A; Table I). However, SKOV-3 cells did not 
A

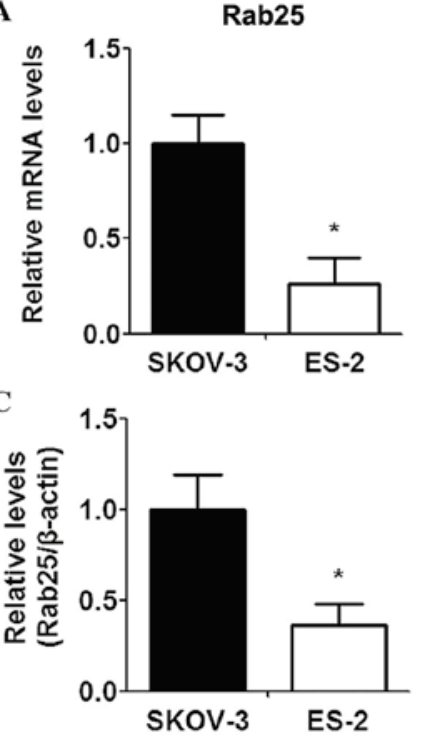

B

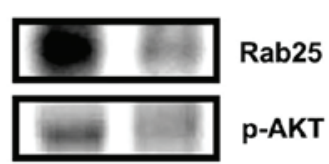

AKT

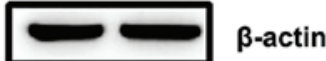

SKVO-3 ES-2

D

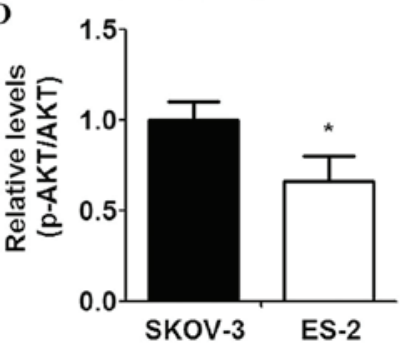

Figure 1. Cisplatin-resistant ovarian cancer cells show overexpression of Rab25 and activation of the phosphoinositide 3-kinase/AKT pathway. (A) mRNA expression levels of Rab25 in SKOV-3 (cisplatin-resistant) and ES-2 (cisplatin-sensitive) cells were measured using reverse transcription quantitative polymerase chain reaction. (B) SKOV-3 and ES-2 cells were plated in six-well plates with $10^{6}$ cells/well and harvested to prepare cell lysates. Western blot analysis was used to analyze Rab25, p-AKT and total AKT expression. $\beta$-actin was used as a loading control. (C and D) Quantification of several immunoblots, similar to the one shown in (B), using Multi Gauge V3.2 software. Values are presented as the mean \pm standard error of the mean $(\mathrm{n}=3)$. ${ }^{*} \mathrm{P}<0.05$ compared with the SKOV-3 cell line. mRNA, messenger RNA; p-AKT, phosphorylated AKT.

A
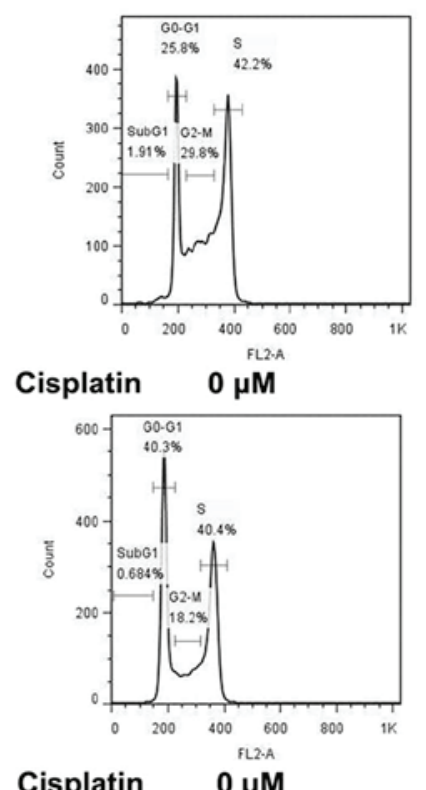

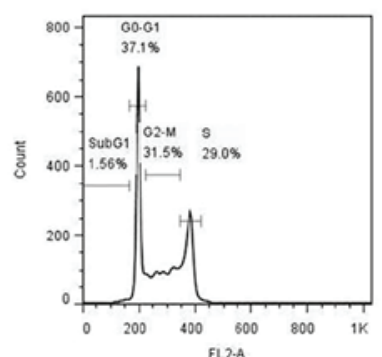

$10 \mu \mathrm{M}$

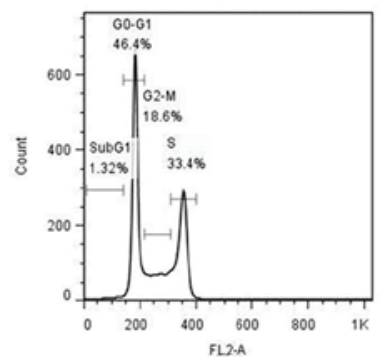

$10 \mu \mathrm{M}$

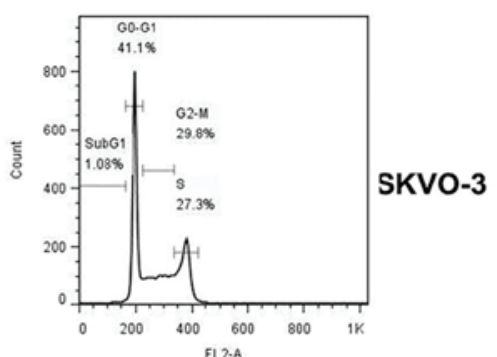

$100 \mu \mathrm{M}$

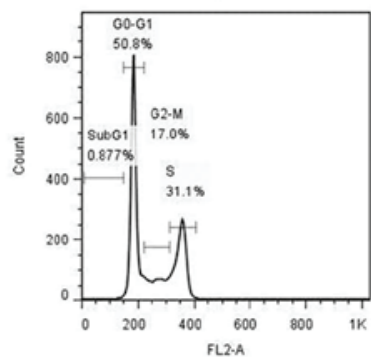

$100 \mu \mathrm{M}$

B

SKVO-3

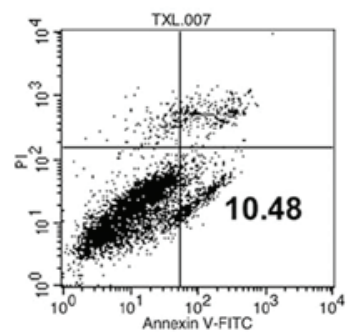

Cisplatin

$10 \mu \mathrm{M}$

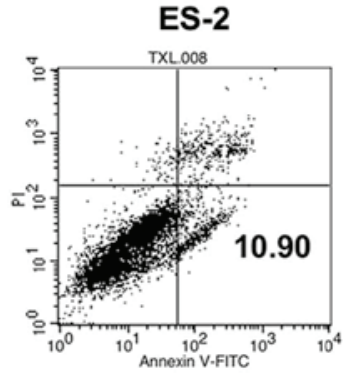

$10 \mu \mathrm{M}$

Figure 2. G1-phase arrest in SKVO-3 cells is lower than that in ES-2 cells. Cells $\left(5 \times 10^{6}\right)$ were cultured in a 6-cm dish. (A) Flow cytometric analysis of the cell cycle in SKVO-3 and ES-2 cells following incubation with a series of cisplatin concentrations for $2 \mathrm{~h}$. (B) Flow cytometric analysis of apoptotic cells following incubation with $10 \mu \mathrm{M}$ cisplatin for $2 \mathrm{~h}$. Experiments were performed in triplicate. FITC, fluorescein isothiocyanate; PI, propidium iodide. 
Table I. Cisplatin-induced G1-phase arrest in SKVO 3 cells is lower than that in ES-2 cells.

\begin{tabular}{|c|c|c|c|c|c|c|}
\hline \multirow[b]{2}{*}{ Phase/Cisplatin } & \multicolumn{3}{|c|}{ SKVO-3 } & \multicolumn{3}{|c|}{ ES-2 } \\
\hline & $0 \mu \mathrm{M}$ & $10 \mu \mathrm{M}$ & $100 \mu \mathrm{M}$ & $0 \mu \mathrm{M}$ & $10 \mu \mathrm{M}$ & $100 \mu \mathrm{M}$ \\
\hline SubG1 (\%) & $1.88 \pm 0.2$ & $1.55 \pm 0.35$ & $1.11 \pm 0.76$ & $1.51 \pm 1.30$ & $16.7 \pm 1.81$ & $1.75 \pm 0.35$ \\
\hline G0/G1 (\%) & $26.7 \pm 2.03^{\mathrm{a}}$ & $37.3 \pm 0.9^{\mathrm{a}}$ & $40.7 \pm 1.69^{\mathrm{a}}$ & $39.7 \pm 2.56$ & $46.7 \pm 3.91$ & $51.3 \pm 1.45$ \\
\hline S (\%) & $41.9 \pm 1.95$ & $28.0 \pm 3.9$ & $30.5 \pm 2.19$ & $38.7 \pm 0.61$ & $29.6 \pm 1.88$ & $31.1 \pm 2.16$ \\
\hline G2/M (\%) & $31.4 \pm 2.74$ & $31.4 \pm 4.67$ & $30.2 \pm 1.07$ & $19.5 \pm 1.49$ & $18.4 \pm 1.87$ & $17.5 \pm 0.83$ \\
\hline
\end{tabular}

Mean \pm standard deviation $(\mathrm{n}=3)$. ${ }^{\mathrm{a}} \mathrm{P}<0.05$ vs. ES-2 cells.

A

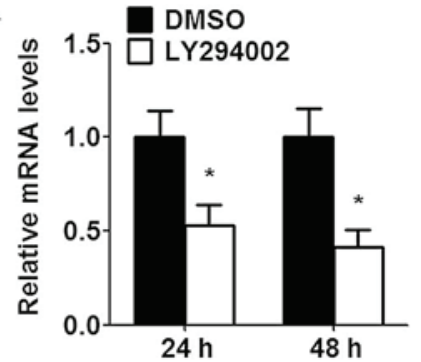

B

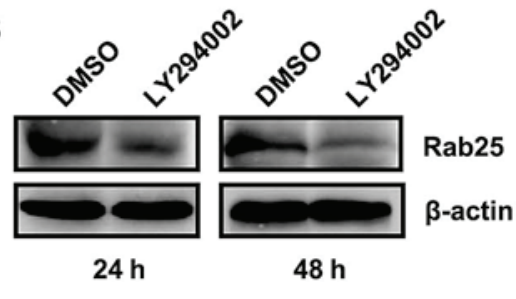

C

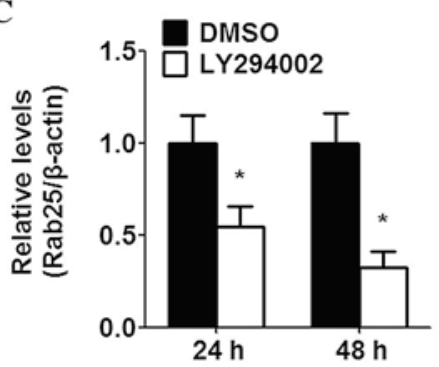

Figure 3. Decreased expression of Rab25 following treatment with Rab25 inhibitor LY294002 in SKOV-3 ovarian cancer cells. (A) Reverse transcription quantitative polymerase chain reaction analysis of Rab25 gene expression in SKOV-3 cells in the presence or absence of LY294002 (10 $\mu \mathrm{M})$ at 24 and $48 \mathrm{~h}$. (B) Western blot analysis of Rab25 protein expression following LY294002 treatment. (C) Optical densities of Rab25 protein bands were analyzed using Multi Gauge V3.2 software and normalized relative to the $\beta$-actin loading control. Values are presented as the mean \pm standard error of the mean ( $\mathrm{n}=3$ ). ${ }^{*} \mathrm{P}<0.05$ vs. DMSO. mRNA, messenger RNA; DMSO, dimethyl sulfoxide.

A

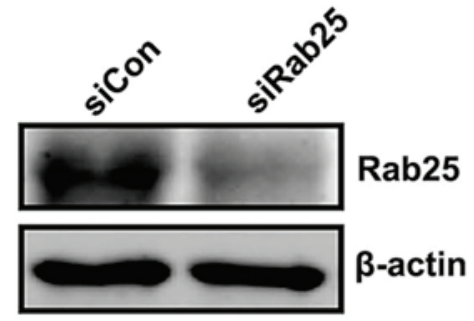

B

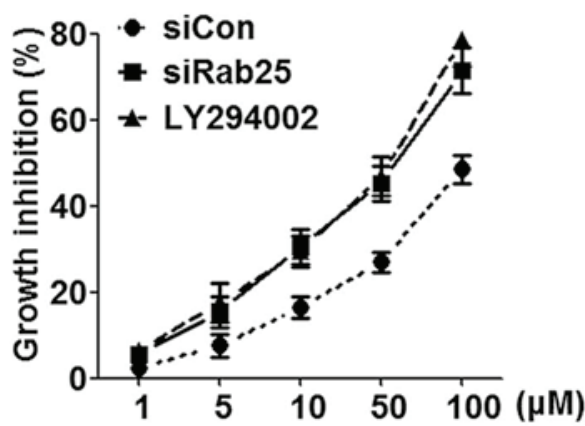

C

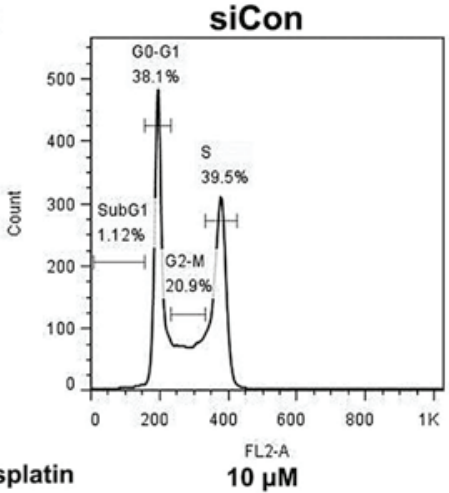

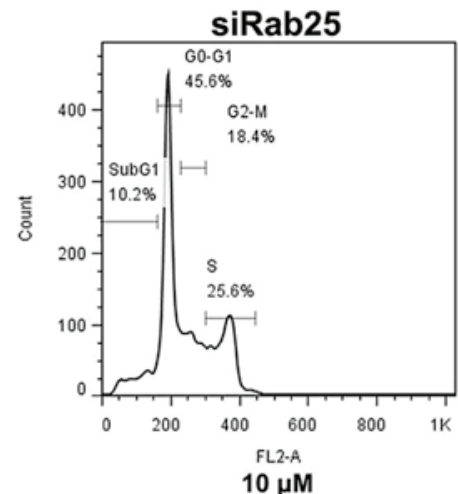

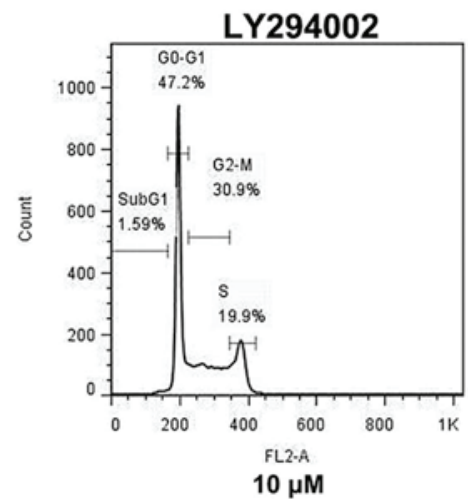

Figure 4. Inhibition of the phosphoinositide 3-kinase/AKT pathway or Rab25 silencing increases the sensitivity of SKOV-3 cells to cisplatin. (A) SKOV-3 cells were transfected with siCon or siRab25 and incubated for $48 \mathrm{~h}$. Western blot analysis was then used to determine Rab25 protein expression levels. (B) SKOV-3 cells were treated with concentrations $(1,5,10,50$ and $100 \mu \mathrm{M})$ of cisplatin in combination with transfection of siCon, siRab25 or treated with the Rab inhibitor LY294002 and incubated for $48 \mathrm{~h}$. MTT assay was then used to determine cell viability. Cell growth inhibition rate was calculated as follows: Inhibition rate $=(1-\mathrm{Absorbance}$ value experiment group/Absorbance value control group $) \times 100$. Values are presented as the mean \pm standard error of the mean $(n=3)$. (C) Flow cytometric analysis of the cell cycle of SKOV-3 cells treated with $10 \mu \mathrm{M}$ cisplatin in combination with transfection of siCon, siRab25 or LY294002. Experiments were performed in triplicate. siCon, control small interfering RNA (siRNA); siRab25, Rab25-specific siRNA. 
respond to cisplatin treatment and the number of cells in G1-phase was not significantly altered (Fig. 2A; Table I). The apoptotic rates of the two cell lines showed no significant difference from each other (Fig. 2B). These data indicated that deficiency in G1-phase cell cycle arrest was induced by cisplatin rather than apoptosis and therefore, the lack of SKOV-3 cells in G1-pase may be due to cisplatin resistance.

Inhibition of the PI3K/AKT pathway downregulates Rab25 expression levels. In order to investigate whether Rab25 was associated with the PI3K/AKT pathway, SKOV-3 cells were treated with the PI3K/AKT inhibitor LY294002. Following treatment with $10 \mu \mathrm{M}$ LY294002 for 24 and 48 h, Rab25 mRNA expression in SKOV-3 cells was decreased by $\sim 46.7$ and $58.4 \%$, respectively (Fig. 3A). Subsequently, Rab25 protein expression levels were determined using western blot analysis. The results demonstrated that LY294002 treatment markedly suppressed Rab25 protein expression; this therefore indicated that inhibition of the PI3K/AKT pathway downregulated Rab25 protein expression (Fig. 3B and C).

Knockdown of Rab25 or inhibition of the PI3K/AKT pathway increases the sensitivity of SKOV-3 cells to cisplatin. In the present study, a Rab25-specific siRNA was used to block the expression of ubiquitously-produced Rab25 in SKOV-3 cells. Western blot analysis revealed that protein levels of Rab25 were significantly decreased (by 70.2\%) following siRab25 transfection as compared with those of the control groups (Fig. 4A). In order to determine whether Rab25 or the PI3K/AKT pathway were involved in the decreased chemosensitivity of cisplatin-resistant cells, the cell growth inhibiting effect of siRab25 or PI3K/AKT signaling inhibitors in combination with cisplatin was measured. An MTT assay demonstrated that siRab25 as well as LY294002 decreased the $\mathrm{IC}_{50}$ values of cisplatin on SKOV-3 cells (Fig. 4B). In addition, Rab25 gene silencing or LY294002 treatment increased the G1-phase cell cycle arrest induced by cisplatin (Fig. 3C). In conclusion, these results indicated that elevation of Rab25, via activation of the PI3K/AKT pathway, may be the mechanism for cisplatin resistance of ovarian cancer cells.

\section{Discussion}

Rab25, a Rab11 subfamily protein, was previously reported to be expressed in all eukaryotes, where it shared a conserved mechanism of regulation (12). Rab25 has been suggested to have a comparable function to that of Rab11; in addition, Rab25 was reported to be spatially and functionally associated with the regulation of apical-to-basolateral transcytosis in polarized epithelial cells, indicating that Rab25 was an important regulator of polarized cell surface composition (18). Rab25 was shown to enhance the invasive ability of cells due to its epithelial cell polarity modulatory characteristic, therefore suggesting that Rab25 dysregulation may have a role in tumorigenesis (14). Previous studies have demonstrated that Rab25 controlled tumor progression, aggressiveness and potentially chemosensitivity $(13,19-21)$; furthermore, Rab25 was amplified at the DNA level and overexpressed at the RNA level in ovarian cancers (13).
Knockdown of RAB25 promotes autophagy and inhibits cell growth in ovarian cancer (15). The results of the present study were concurrent with the hypothesis that Rab25, as an oncogene, contributed to the aggressiveness of ovarian cancer. In addition, the present study demonstrated that constitutive overexpression of Rab25 due to increased activation of PI3K/AKT signaling in SKOV-3 cells resulted in cisplatin resistance, a severe obstacle for the successful treatment of ovarian cancer. Elucidating the molecular mechanism underlying cisplatin resistance is critical for improving sensitivity to chemotherapeutic agents. It is understood that chemoresistance is due to dysregulation of the balance between the pathways of cellular survival and apoptosis as well as enhanced drug clearance, enhanced detoxification and reduced drug efficacy due to increased DNA repair (22). A previous study has reported that inhibition of the PI3K/AKT pathway may enhance chemosensitivity of resistant ovarian cancers, more prominently in tumors with a high PI3K/AKT activity profile (10). The present study revealed that suppression of the PI3K/AKT pathway by LY294002 reduced Rab25 expression, therefore indicating that Rab25 was a key effector molecule of the PI3K/AKT pathway in cisplatin resistance. Furthermore, knockdown of Rab25 using siRNAs or inhibition of PI3K/AKT signaling increased the sensitivity of SKOV-3 cells to cisplatin via augmentation of G1-phase cell cycle arrest, which was thought to contribute to the sensitization of cells to cisplatin.

In conclusion, the results of the present study confirmed the tumorigenic role of RAB25 in ovarian cancer cells and suggested a novel role of Rab25 in cisplatin resistance. In addition, the results demonstrated that inhibition of Rab25 and the PI3K/AKT pathway sensitized ovarian cancer cells to cisplatin, providing a potential novel adjuvant therapy in combination with cisplatin.

\section{Acknowledgements}

The present study was supported by a grant from the National Natural Science Foundation of China (no. 81060215).

\section{References}

1. Kim A, Ueda Y, Naka T and Enomoto T: Therapeutic strategies in epithelial ovarian cancer. J Exp Clin Cancer Res 31: 14, 2012.

2. Colombo N, Van Gorp T, Parma G, et al: Ovarian cancer. Crit Rev Oncol Hematol 60: 159-179, 2006.

3. Wrzeszczynski KO, Varadan V, Byrnes J, et al: Identification of tumor suppressors and oncogenes from genomic and epigenetic features in ovarian cancer. PloS One 6: e28503, 2011.

4. Holschneider CH and Berek JS: Ovarian cancer: epidemiology, biology, and prognostic factors. Semin Surg Oncol 19: 3-10, 2000.

5. Shih Ie M and Davidson B: Pathogenesis of ovarian cancer: clues from selected overexpressed genes. Future Oncol 5: 1641-1657, 2009.

6. Nelson AE, Francis JE and Zorbas H: Population screening and early detection of ovarian cancer in asymptomatic women. Aust NZ J Obstet Gynaecol 49: 448-450, 2009.

7. Trimbos JB,Parmar M, Vergote I, et al; International Collaborative Ovarian Neoplasm 1 and the European Organisation for Research and Treatment of Cancer Collaborators-Adjuvant Chemotherapy in Ovarian Neoplasm. International collaborative ovarian neoplasm trial 1 and adjuvant chemotherapy in ovarian neoplasm trial: two parallel randomized phase III trials of adjuvant chemotherapy in patients with early-stage ovarian carcinoma. J Natl Cancer Inst 95: 105-112, 2003.

8. Aabo K, Adams M, Adnitt P, et al: Chemotherapy in advanced ovarian cancer: four systematic meta-analyses of individual patient data from 37 randomized trials. Advanced ovarian cancer trialists' group. Br J Cancer 78: 1479-1487, 1998. 
9. Markman M: Optimizing primary chemotherapy in ovarian cancer. Hematol Oncol Clin North Am 17: 957-968, 2003.

10. Ali AY, Farrand L, Kim JY, et al: Molecular determinants of ovarian cancer chemoresistance: new insights into an old conundrum. Ann NY Acad Sci 1271: 58-67, 2012.

11. Mitra S, Cheng KW and Mills GB: Rab25 in cancer: a brief update. Biochem Soc Trans 40: 1404-1408, 2012.

12. Agarwal R, Jurisica I, Mills GB and Cheng KW: The emerging role of the RAB25 small GTPase in cancer. Traffic 10: 1561-1568, 2009.

13. Cheng KW, Lahad JP, Kuo WL, et al: The RAB25 small GTPase determines aggressiveness of ovarian and breast cancers. Nat Med 10: 1251-1256, 2004.

14. Caswell PT, Spence HJ, Parsons M, et al: Rab25 associates with alpha5betal integrin to promote invasive migration in 3D microenvironments. Dev Cell 13: 496-510, 2007.

15. Liu Y, Tao X, Jia L, et al: Knockdown of RAB25 promotes autophagy and inhibits cell growth in ovarian cancer cells. Mol Med Rep 6: 1006-1012, 2012.
16. Fan Y, Xin XY, Chen BL and Ma X: Knockdown of RAB25 expression by RNAi inhibits growth of human epithelial ovarian cancer cells in vitro and in vivo. Pathology 38: 561-567, 2006.

17. Wang $\mathrm{H}, \mathrm{Li} \mathrm{H}, \mathrm{ZuoM}$, et al: $\mathrm{Lx} 2-32 \mathrm{c}$, a novel taxane and its antitumor activities in vitro and in vivo. Cancer Lett 268: 89-97, 2008.

18. Goldenring JR and Nam KT: Rab25 as a tumour suppressor in colon carcinogenesis. Br J Cancer 104: 33-36, 2011.

19. Cheng KW, Lahad JP, Gray JW and Mills GB: Emerging role of RAB GTPases in cancer and human disease. Cancer Res 65: 2516-2519, 2005

20. Casanova JE, Wang X, Kumar R, et al: Association of Rab25 and Rab11a with the apical recycling system of polarized Madin-Darby canine kidney cells. Mol Biol Cell 10: 47-61, 1999.

21. Wang X, Kumar R, Navarre J, Casanova JE and Goldenring JR: Regulation of vesicle trafficking in madin-darby canine kidney cells by Rab1la and Rab25. J Biol Chem 275: 29138-29146, 2000.

22. Fraser M, Leung B, Jahani-Asl A, Yan X, Thompson WE and Tsang BK: Chemoresistance in human ovarian cancer: the role of apoptotic regulators. Reprod Biol Endocrinol 1: 66, 2003. 